\title{
Marker-3D Measurement Versus Traditional Radiograph Measurement in the Treatment of Tibiofibular Fracture Using Taylor Spatial Frame
}

Qixin Liu

Graduate College of Tianjin Medical University

Yanshi Liu

the First Affiliated Hospital of Xinjiang Medical University

Hong Li

Zigong Fourth People's Hospital

Xuefei Fu

Anhui No.2 Provincial People's Hospital

Xingpeng Zhang

Shanghai Pudong New Area People's Hospital

Sida Liu

Tianjin University

Jinli Zhang

Tianjin Hospital

Tao Zhang ( $\square$ ztorthopaedic@163.com )

Tianjin Hospital

\section{Research Article}

Keywords: External fixation, Taylor Spatial Frame, Three-dimensional reconstruction, Tibiofibular fracture

Posted Date: July 15th, 2021

DOI: https://doi.org/10.21203/rs.3.rs-712196/v1

License: (1) (1) This work is licensed under a Creative Commons Attribution 4.0 International License. Read Full License 


\section{Abstract}

Background: The Taylor Spatial Frame (TSF) has been widely used for tibiofibular fractures. However, traditional radiograph measurement method is complicated and the reduction accuracy is not high enough for correcting residual deformities. We proposed the marker-3D measurement method to solve these problems. This study aimed to compare the reduction accuracy of the traditional radiograph measurement method and the marker-3D measurement method in tibiofibular fracture treated with TSF.

Methods: From January 2016 to June 2019, A retrospective analysis was performed based on the patients with tibiofibular fracture treated with TSF in our department. 41 patients were qualified for this study, including 21 patients in the marker-3D measurement group (experimental group) and 20 patients in the traditional radiograph measurement group (control group). In the experimental group, CT scan was performed for 3D reconstruction with 6 markers installed on the TSF, to determine the adjusting plan. In the control group, the anteroposterior (AP) and lateral radiographs were performed for the deformity parameters. X-rays were taken to measure the residual deformities after correction.

Results: All patients reached functional reduction. The residual displacement deformity (RDD) in AP radiograph was $0.5(0,1.72) \mathrm{mm}$ in experimental group and $1.74(0.43,3.67) \mathrm{mm}$ in control group. The residual angle deformity $(\mathrm{RAD})$ in AP radiograph was $0(0,1.25)^{\circ}$ in experimental group and $1.25(0.62$, $1.95)^{\circ}$ in control group. As to the Lateral radiograph, the RDD was $0(0,1.22) \mathrm{mm}$ in experimental group and $2.02(0,3.74) \mathrm{mm}$ in control group. The RAD was $0(0,0)^{\circ}$ in experimental group and $1.42(0,1.93)^{\circ}$ in control group. Significant differences in all above comparisons were found between the groups (AP radiograph $\mathrm{RDD}: \mathrm{P}=0.024, \mathrm{RAD}: \mathrm{P}=0.020$; Lateral radiograph $\mathrm{RDD}: \mathrm{P}=0.016, \mathrm{RAD}: \mathrm{P}=0.004)$.

Conclusions: Both groups achieved satisfactory fracture reduction. However, the residual deformities in the experimental group were significantly smaller. This study proved that the marker-3D measurement method could further improve the reduction accuracy.

\section{Background}

Tibia fracture is a common type fracture of the long bone fractures, accounting for about $17-21 \%$ of lower limb fractures, the average healing time ranges from 15 weeks to 40 weeks, and the healing time of open fractures could be extended or even not healed ${ }^{1,2}$. External fixation is able to play an important role in this type fracture. External fixation can help to retain the microenvironment of fracture healing and achieve good results in the treatment of bone nonunion, osteomyelitis and other diseases ${ }^{3-6}$. External fixation had been described for the treatment of transverse patellar fractures as early as $1843^{7}$. However, because of the limited theory and technology at that time, external fixation treatment frequently received dissatisfied efficacy and complications. Until Professor llizarov demonstrated the tension stress effect on tissue regeneration and then invented the llizarov external fixator, the external fixation technology was greatly developed and cured many patients with fracture nonunion, deformities, and osteomyelitis ${ }^{8}$. However, with the use of Ilizarov system, special frame structure needs to be designed for 
specific situation, and the residual deformities are difficult to be corrected. Mastering the llizarov system requires a steep learning process ${ }^{9}$.

In the 1990s, Charles Taylor and his brother Harold developed a new type of external fixator, Taylor Spatial Frame (TSF), based on the Stewart platform and Ilizarov external fixator. The basic TSF structure consists of two complete or incomplete rings connected by six telescopic struts to a universal joint on each end. The spatial structure between the two rings can be freely changed by adjusting the length of any struts. By importing the measured parameters into the supporting computer software, TSF can correct the deformities of limbs simultaneously such as angulation, translation, rotation and shortening ${ }^{10}$. Compared with llizarov external fixator, TSF can correct the deformity in three-dimensional space by only adjusting the length of struts, without the change of fixator's configurations. The installation is simpler and the learning process is shorter than llizarov external fixator ${ }^{9}$. Nowadays, TSF has been widely used in orthopedic surgeries because of its advantages.

However, when using an external fixator, deformity parameters need to be manually measured on radiographs, which may contain measurement errors ${ }^{11,12}$. Radiographs cannot obtain accurate information about the limb's axial view: deformity parameters related to the rotation has to be estimated by physical examinations ${ }^{13}$. The measurement of the parameters is greatly disturbed by the subjective error of the measurer ${ }^{14}$. The above drawbacks often lead to poor alignment of the fracture after reduction. As a result, the deformity may need to be corrected multiple times and lead to the delay of treatment ${ }^{15}$. Furthermore, lower limb malalignment is an independent risk factor for knee osteoarthritis, the load distribution of the knee joint surface will be affected by the malalignment in the lower limbs, especially varus alignment increases the risk of tibiofemoral osteoarthritis and medial meniscus lesions ${ }^{16-19}$. Better alignment after fracture reduction is conducive to the long-term prognosis of patients. Therefore, this study aimed to introduce a new method to improve the accuracy of fracture reduction with TSF.

With the development of imaging technology, 3D reconstruction technology has been widely used in medicine ${ }^{20-22}$. The 3D model can be obtained by the two-dimensional grayscale data from CT scan. In fracture cases, the 3D reconstruction technology provides the axial information of the patient's, which traditional X-Ray cannot present but is extremely important to improve the accuracy of fracture reduction. The fracture line, the articular surface, and the joint cavity can be observed objectively in 3D reconstruction, which helps surgeons to understand the fracture comprehensively.

In previous studies, installing markers on the rings were introduced into the process of CT scan and 3D reconstruction ${ }^{23}$. In this study, we proposed a new marker-3D measurement method and applied to TSF treatment. The adjustment plans of struts can be automatically generated with the help of self-developed software, avoiding the manual measurement of deformity parameters.

The purpose of this study was to compare the reduction accuracy of the traditional radiograph measurement method and the marker-3D measurement method in tibia fractures treated with TSF after 
adjusting by comparing:(1) the residual displacement deformity (RDD) in anteroposterior (AP) and lateral radiographs between the two methods; (2) the residual angle deformity (RAD) in anteroposterior (AP) and lateral radiographs between the two methods.

\section{Methods}

\section{Study design and patients}

The cases with tibia fractures, treated with TSF in Tianjin Hospital from January 2016 to June 2019, were retrospectively analyzed. The inclusion criteria were: (1) comminuted fracture (AO/Asif classification C3); (2) compound fractures (Gustilo type II / III); (3) the postoperative follow-up time of patient was $\geq 6$ months. Exclusion Criteria were: (1) Patients with bilateral tibia fractures (unable to provide the mirror image of the contralateral three-dimensional reconstruction image); (2) patients unable to cooperate with regular follow-up. Finally, 41 patients were included in the study. There were 21 patients in the marker-3D measurement group (experimental group) and 20 patients in the traditional radiograph measurement group (control group).

\section{Measurement methods}

All surgeries were performed by the same surgeon and all following procedures were performed by the same processor, to make sure that all treatment followed with the same standard. All patients were installed with TSF by the following methods: first fixing the frame to the bone segment, the struts were in a sliding state, the fracture was preliminarily reduced by moving the rings under the $\mathrm{C}$-arm and then the struts were locked, deformities would be corrected by adjusting TSF struts after the operation.

\section{Traditional radiograph measurement}

The standard radiographs (AP and lateral radiographs, including proximal and distal joints as much as possible) of patients were measured after the operation. The X-ray images were imported into computer for measurement to determine the deformity parameters (Figure 1). The proximal bone segment was used as the fixed end, and the distal bone segment was determined as the free movement end. The midpoint of the proximal fracture line was taken as the center of rotation of deformation (CORA).

\subsection{Anteroposterior measurement}

(1) Point 0 was the center of the proximal ring by finding the longest diameter of the ring; (2) a1 was defined as the angle between the mechanical axial of the proximal and distal bone segments; (3) S1 was recorded as the internal or external displacement distance of the distal bone segment. Selecting a starting point on the proximal bone segment (it was easier to identify by choosing the protuberant tip on the bone). Meanwhile, the corresponding point of the anatomical relationship was specified on the distal bone segment. Then S1 could be determined; (4) L1 was marked as the left or right offset distance of the proximal ring center relative to the center of the tibia. Because the TSF system defaulted that the proximal ring was perpendicular to the proximal bone segment, so a perpendicular line from point 0 to 
the proximal tibial mechanical axis was made, and the distance from point $\mathrm{O}$ to the mechanical axis was L1. (Figure 1a).

\subsection{Lateral measurement}

(1) a2 was recorded as the tension or flexion angle of the distal bone segment; (2) the anterior or posterior displacement distance of the distal bone segment was recorded as S2; (3) the anterior or posterior offset distance between the center of the proximal ring and the center of the tibia was recorded as L2.(Figure 1b).

\subsection{Axial measurement}

(1) T was recorded as the shortening or separation displacement of the distal bone(Figure 1a); (2) the rotation deformity of the limb was determined by physical examination, due to conventional X-ray images didn't contain the patient's axial information; (3) the rotation offset angle of the proximal ring could not be measured from the X-ray, which was only set by the doctor's experience; (4) the axial distance from the proximal ring to the fractured segment could be measured from the AP X-ray, which was denoted as L3( Figure 1a).

Finally, the above parameters were inputted into the TSF supporting software. According to the severity of the trauma, we would have the patients planned in different treating period, and then drafted the adjusting plan of the struts.

\section{Marker-3D measurement method}

The marker,3D reconstruction, and designated software were applied in this study to reduce the measurement error while determining the adjusting plan.

\subsection{Marker}

The marker was a composite structure, which was composed of aluminum alloy marker ball and photosensitive resin connecting rod (Figure 2). A set of markers was used for measurement, the size and material of each marker were the same.

\subsection{D Reconstruction}

The markers were mounted on the rings with three on the proximal and the others on the distal. The markers were distributed on each ring as evenly as possible (120 degrees). Every patient in the marker-3D measurement group was scanned by CT (GE Optima, CT66) on both lower limbs for 3D reconstruction. The following models were generated: the 3D model of the proximal bone of the affected limb (Model Proximal), the 3D model of the distal bone of the affected limb (Model Distal), the 3D mirror model of healthy limb bone (Model Reference), and the 3D model of external fixation (Model Frame), and the 3D model of Marker Balls (Model Marker Balls)(Figure 3). The 3D mirror model of healthy limb was used for 
registration (According to the principle of mirror symmetry of the human body, the mirror image of the bone could be used as the registration standard of the affected side bone ${ }^{23,24}$ )

\subsection{Preparation in software}

It was considered that the proximal bone-ring and the distal bone-ring of the broken limb formed a rigid part respectively. We designed and developed a 3D reduction software for the measurement of TSF (Figure 4). The detailed marker locations on the ring needed to be inputted into the reduction software. The reduction software could recognize the spatial position of the marker balls automatically and determine the initial position and the pose of the two fixed rings. During the fracture reduction, the software could automatically record the change in position and pose.

\subsection{Simulated fracture reduction in the software}

(1) The reconstructed 3D models and the information of the frame and markers were imported into the

3D fracture reduction software for simulation; (2) the bone was operated to simulate the reduction movement in the graphic interface of the software. The protuberance tip on the bone segment and the feature point on the joint were used as the reference points, the software took the Model Proximal as the fixed end and registered the Model Distal with Model Reference to achieve fracture reduction directly; besides, it was also possible to add multiple reduction intermediate points according to requirements. "traction-rotation-alignment" was the motion path of the bone to ensure the safety of reduction; (3) The software could automatically generate the reduction path of the free movement end with avoiding the interaction between the bone segments, and generated the reduction motion simulation animation for the reference of clinicians according to the initial and final position and posture of the fixed ring; (4) the software determined the relative position and the posture changes of the two fixed rings according to step 3. Then the software calculated the length changes of the six struts with the Stewart mechanism kinematics algorithm, and generated the strut 's adjustment plan (electronic prescription) of TSF. The schematic diagram was shown in Figure 5. Patients would adjust the struts according to the adjustment plan (Figure 6).

\section{Outcome evaluation}

In the end of adjusting, all patients took standard X-rays of the affected side bone, and then we compared the differences in the residual deformities of the two groups according to radiographs.

The displacement deformity was the distance of the inward/outward movement in AP X-ray or forward/backward movement in lateral X-ray of the distal bone segment relative to the proximal bone segment. The angle deformity was the angle of varus/valgus in AP X-ray or extension/flexion in lateral Xray of the mechanical axis of the distal bone segment relative to the mechanical axis of the proximal bone segment.

\section{Statistical analysis}


SPSS 22 (IBM Inc., New York, USA) was used for statistical analysis. The comparison between age was conducted by Student's $t$ test, the age was represented by ' $\mathrm{x} \pm \mathrm{s}$; the comparison of Categorical data between groups was conducted by chi-square test. The measurement data of abnormal distribution (residual deformities) was expressed as M (P25, P75), Mann-Whitney $\mathrm{U}$ test were used for comparison between groups. The significant level in this study was considered as $\mathrm{P} \leq 0.05$.

\section{Results}

\section{General information in two groups}

41 patients were included in this study. All patients receive follow-up for more than 6 months after surgery. The experimental group comprised of 15 males and 6 females, with an average age of $49.5 \pm 14.8$ years, ranging from 18 to 73 years. There were 13 cases of compound fractures (Gustilo classification Type II) and 8 cases of compound fractures (Gustilo classification Type III). The control group comprised of 17 males and 3 females, aged 19-76 years with an average of $47.6 \pm 14.3$ years. The BMI of the experimental group and control group was $23.7 \pm 2.4 \mathrm{~kg} / \mathrm{m}^{2}$ and $23.4 \pm 2.2 \mathrm{~kg} / \mathrm{m}^{2}$. There were 14 cases of compound fractures (Gustilo classification Type II) and 6 cases of compound fractures (Gustilo classification Type III). There were no statistical differences between the two groups in terms of gender, age, and fracture type( $P>0.05)$ (Table 1$)$.

\section{Residual displacement deformity (RDD)}

In the experimental group, the RDD in AP radiograph was $0.5(0,1.72) \mathrm{mm}$; in the control group, the RDD in AP radiograph was $1.74(0.43,3.67) \mathrm{mm}$. The RDDs in AP X-ray between the two groups were significantly different $(P=0.024)$;

In the experimental group, the RDD in lateral radiograph was $0(0,1.22) \mathrm{mm}$; in the control group, the RDD in lateral radiograph was $2.02(0,3.74) \mathrm{mm}$. The RDDs in lateral X-ray between the two groups were significantly different $(P=0.016)$ (Table 2$)$.

\section{Residual angular deformity (RAD)}

In the experimental group, the RAD in AP radiograph were respectively $0(0,1.25)^{\circ}$; in the control group, the RAD in AP radiograph were $1.25(0.62,1.95)^{\circ}$. The RADs in AP X-ray between the two groups were significantly different $(P=0.020)$.

In the experimental group, the RAD in lateral radiograph were respectively $0(0,0)^{\circ}$; in the control group, the RAD in lateral radiograph were $1.42(0,1.93)^{\circ}$. The RADs in AP X-ray between the two groups were significantly different $(P=0.004)$ (Table 2$)$.

The residual deformities of displacement and angle in the experimental group were smaller than those in the control group in the AP and lateral X-ray. This result proposed that the reduction accuracy was higher and the fracture alignment was better with 3D-marker measurement method. 


\section{Discussion}

We proposed a method which could automatically identify the spatial configuration of the frame by adding markers to TSF to reduce the measurement error and improve the reduction accuracy. According to table 2, the residual deformities were significantly smaller in experimental group than control group $(P<0.05)$. These indicated that the marker-3D measurement method could further improve the accuracy and reduce the residual deformities comparing to traditional $\mathrm{X}$-ray measurement method.

Compared with the traditional measurement that uses X-Ray imaging for planning, fractures reconstructed with the marker-3D measurement method showed better reduction accuracy, which could come from reduced measurement error and the reduction of axial rotational deformity. Traditional measurement method requires multiple measurements and adjustments after surgery to achieve satisfactory reduction ${ }^{15}$. In most of the patients with 3D measurement method, the satisfactory reductions could be achieved by the initial adjustment plan.

Other orthopedic surgeons had done a lot of research in these areas. Simpson et al used CT images for $3 \mathrm{D}$ reconstruction to perform virtual surgery ${ }^{14}$. He introduced a tracking stylus to digitize the connection holes as reference points on the TSF ring, or used the information of the bone surface for registration. This method avoided the measurement of parameters to reduce measurement errors, but the result was greatly interfered by the choice of connecting holes. The positions of these connecting holes may be affected by the installation of Kirschner wires and struts, resulting in the failure to find a suitable connection hole as a reference point, and metal artifacts would also affect the imaging.

Similar to our study, Tang et al designed a hexapod automatic fracture reduction device, similar to the Stewart platform, and then tested in animal models ${ }^{23}$. With the help of three-dimensional reconstruction from CT scan, 12 marker balls were used to replace 12 screw bolts, and the hinger's length was directly identified by software, then the electric hinges automatically reduced the fracture. However, this automatic reduction may not be able to consider the soft tissue, the blood supply, and the shape of fracture during the process of reduction, the bone segments may even get stuck during the process of reduction.

$\mathrm{Du} \mathrm{H}$ et al improved the above device for clinical usage and redesigned the device as a combination of a positioning unit, a reduction unit, and a control center ${ }^{25}$. Four non-special marking points of the positioning unit were used for registration to obtain the length of the struts. They introduced a seriesparallel configuration to convert the 6-DOF movements of the hexapod mechanism into relevant movements of two holders, which prevented the device from jamming during resetting and improved the portability of the device. However, there were several disadvantages in this design. First, the positioning unit was composed of four parts which were complicated to disassemble and could lose precision during the process. Second, the various customized rings and devices may limit the clinical application of the device. The Last but not the least, this method only provided a temporary reduction method during 
surgery, it still needs effective external fixation or internal fixation after the reduction, and the device also needed to be customized, which was not suitable for clinical promotion.

In this study, to improve the ease of use, we used standard TSF, designed a marker, and developed a software. The markers could be installed freely, and the software was able to automatically identify the position of the marker balls. With the help of the markers, the processes of measurement could be finished automatically, which helped avoiding manual measurement errors. In addition, a set of computerassisted TSF reduction software based on CT images was introduced. The position and posture of bone segments were automatically recognized by the markers. The adjustment plan of external fixation for fracture reduction was obtained through the final relative displacement transformation matrix.

With the help of CT data, the proposed marker-3D measurement method could accurately obtain the axial information of the injured limb and generate adjusting plan, compared with the traditional measuring method based on X-ray. In order to ensure the safety of the reduction and to avoid the soft tissue damage around the fracture, the self-developed software was able to examine the path of the fracture reduction process. Two basic principles were used in the reduction process: (1) necessary bone segment traction and rotation need to be applied to avoid the collision of the fracture segments; (2) the bone segments are aligned with minimal movement while avoid overstretching of soft tissue. As a result, the marker-3D measurement method illustrated high reduction accuracy.

Previous studies have proved that the lower limb malalignment will increase the risk of knee OA and medial meniscus lesions ${ }^{16-19}$. The marker-3D measurement method had high reduction accuracy and will be able to effectively improve the alignment comparing to traditional X-ray method. The better the alignment is, the better the patient's long-term prognosis will be. The marker method makes the treatment process intuitive and convenient; it has a wide prospect of application.

The use of markers to achieve automatic measurement also had the following shortcomings: (1) During CT scanning, the metal parts of TSF could produce metal artifacts interfering with the morphology of the $3 \mathrm{D}$ reconstruction of the bone, thereby affecting the accuracy of the reduction; (2) The sample size of this study was relatively small, and larger sample size could help to verify the effectiveness of this study; (3) The surgeons need computer skills and are able to master the use of 3D reconstruction and other software;(4) The radiation load of CT is larger than X-ray.

In the next step, the imaging technology needs to be improved to minimize the metal artifacts in the reconstruction process for improving the accuracy of reduction. Our registration remained as a manual point-to-point registration in this study, how to achieve automatic registration will be one of our next research objectives. In addition, we are planning to use optical trackers and markers to evaluate fractures and fracture reduction more comprehensively ${ }^{26}$. Furthermore, the automatic reduction robot system will be used as our next research direction.

In conclusion, we introduced a marker-3D measurement method that introduced the marker onto the current mainstream TSF for easy installation and simple operating. The marker is simple to install and 
disassemble, and is fully compatible with the current mainstream external fixation instruments. The reconstructed 3D bone model provided axial information of patients and could also help surgeons better understand the mechanism of injury. The marker-3D measurement method is able to improve the accuracy of fracture reduction and avoid manual measurement error in the clinical application of TSF. This method in clinical application is conducive to the patient's rehabilitation and bone healing.

\section{Conclusions}

Both groups achieved satisfactory fracture reduction. However, the residual deformities in the experimental group were significantly smaller. This study proved that the marker-3D measurement method could further improve the reduction accuracy.

\section{Abbreviations}

TSF: Taylor spatial frame

CT: computed tomography

RDD: residual displacement deformity

RAD: residual angle deformity

AP: anteroposterior

3D: three-dimensional

CORA: center of rotation of deformation

6-DOF: 6 degrees of freedom

OA: osteoarthritis

\section{Declarations}

\section{Ethics approval and consent to participate}

All methods in this study were carried out in accordance with the Declaration of Helsinki. This study was approved by the Ethics Committee of Tianjin Hospital. Written informed consent was obtained from all patients for their data to be recorded in our study.

\section{Consent for publication}

Informed consent was obtained from all patients for their data to be published in our study.

\section{Availability of data and materials}


The datasets analysed during the current study are available from the corresponding author on reasonable request.

\section{Competing interests}

The authors declare that they have no competing interests.

\section{Funding}

This study was funded by the grants from the National Natural Science Foundation of China (No.51775367) and the Foundation of Science and Technology Department of Tianjin (No. 18YFSDZC00010). The funding body was involved in the collection, analysis, and interpretation of data by supporting with salary for the time needed. They were not involved in the design or writing the manuscript.

\section{Authors' Contributions}

QXL: Conducted the study. Collected, analyzed and interpreted the data. Wrote the manuscript.

YSL: Interpreted and analyzed the data. Edited the manuscript.

HL: Created and statistical analyzed the data. Edited the manuscript.

XFF: Provided software assistance.

XPZ: Conducted the study and provided the data.

SDL: Conducted the study and provided the data.

JLZ: Planned the project. Reviewed the manuscript.

TZ: Planned the project. Reviewed the manuscript.

Qixin Liu, Yanshi Liu, and Hong Li are contributed equally to this study.

All authors read and approved the final manuscript.

\section{Acknowledgements}

The authors thank the team of Professor Zhi Yang from Capital Medical University and the team of Professor Tao Sun from Tianjin University for their guidance on 3D reconstruction in this research.

\section{References}

1. Milenkovic S, Mitkovic M, Mitkovic M. External fixation of segmental tibial shaft fractures. Eur J Trauma Emerg Surg 2020;46:1123-7. 
2. Corey RM, Park NK, Cannada LK. Segmental Tibia Fractures: An Analysis of Complication and Healing Rates. J ORTHOP TRAUMA 2018;32:296-300.

3. Chavoshnejad P, Ayati M, Abbasspour A, Karimpur M, George D, Remond Y, et al. Optimization of Taylor spatial frame half-pins diameter for bone deformity correction: Application to femur. Proc Inst Mech Eng H 2018;232:673-81.

4. Tsibidakis H, Panou A, Angoules A, Sakellariou VI, Portinaro NM, Krumov J, et al. The Role of Taylor Spatial Frame in the Treatment of Blount Disease. Folia Med (Plovdiv) 2018;60:208-15.

5. Hughes A, Parry M, Heidari N, Jackson M, Atkins R, Monsell F. Computer Hexapod-Assisted Orthopaedic Surgery for the Correction of Tibial Deformities. J ORTHOP TRAUMA 2016;30:e256-61.

6. Fadel M, Hosny G. The Taylor spatial frame for deformity correction in the lower limbs. INT ORTHOP 2005;29:125-9.

7. Hernigou P. History of external fixation for treatment of fractures. INT ORTHOP 2017;41:845-53.

8. Gubin AV, Borzunov DY, Marchenkova LO, Malkova TA, Smirnova IL. Contribution of G.A. Ilizarov to bone reconstruction: historical achievements and state of the art. Strategies Trauma Limb Reconstr 2016;11:145-52.

9. Thiryayi WA, Naqui Z, Khan SA. Use of the Taylor spatial frame in compression arthrodesis of the ankle: a study of 10 cases. J FOOT ANKLE SURG 2010;49:182-7.

10. Paley D. History and Science Behind the Six-Axis Correction External Fixation Devices in Orthopaedic Surgery. Operative Techniques in Orthopaedics 2011;21:125-8.

11. Keshet D, Eidelman M. Clinical utility of the Taylor spatial frame for limb deformities. Orthop Res Rev 2017;9:51-61.

12. Vroemen JC, Dobbe JG, Strackee SD, Streekstra GJ. Positioning evaluation of corrective osteotomy for the malunited radius: 3-D CT versus 2-D radiographs. ORTHOPEDICS 2013;36:e193-9.

13. Taylor JC. Perioperative planning for two- and three-plane deformities. FOOT ANKLE CLIN 2008;13:69-121.

14. Simpson AL, Ma B, Slagel B, Borschneck DP, Ellis RE. Computer-assisted distraction osteogenesis by llizarov's method. Int J Med Robot 2008;4:310-20.

15. Kucukkaya M, Karakoyun O, Armagan R, Kuzgun U. Calculating the mounting parameters for Taylor Spatial Frame correction using computed tomography. J ORTHOP TRAUMA 2011;25:449-52.

16. Tanamas S, Hanna FS, Cicuttini FM, Wluka AE, Berry P, Urquhart DM. Does knee malalignment increase the risk of development and progression of knee osteoarthritis? A systematic review. Arthritis 
Rheum 2009;61:459-67.

17. Sharma L, Song J, Felson DT, Cahue S, Shamiyeh E, Dunlop DD. The role of knee alignment in disease progression and functional decline in knee osteoarthritis. JAMA 2001;286:188-95.

18. Englund M, Felson DT, Guermazi A, Roemer FW, Wang K, Crema MD, et al. Risk factors for medial meniscal pathology on knee MRI in older US adults: a multicentre prospective cohort study. ANN RHEUM DIS 2011;70:1733-9.

19. Andrews M, Noyes FR, Hewett TE, Andriacchi TP. Lower limb alignment and foot angle are related to stance phase knee adduction in normal subjects: a critical analysis of the reliability of gait analysis data. J ORTHOP RES 1996;14:289-95.

20. Van den Berg J, Struelens B, Nijs S, Hoekstra H. Value of three-dimensional computed tomography reconstruction in the treatment of posterior tibial plateau fractures. KNEE 2020;27:3-8.

21. Thakkar SC, Thakkar RS, Sirisreetreerux N, Carrino JA, Shafiq B, Hasenboehler EA. 2D versus 3D fluoroscopy-based navigation in posterior pelvic fixation: review of the literature on current technology. Int J Comput Assist Radiol Surg 2017;12:69-76.

22. Chen HY, Ng LS, Chang CS, Lu TC, Chen NH, Chen ZC. Pursuing Mirror Image Reconstruction in Unilateral Microtia: Customizing Auricular Framework by Application of Three-Dimensional Imaging and Three-Dimensional Printing. PLAST RECONSTR SURG 2017;139:1433-43.

23. Tang P, Hu L, Du H, Gong M, Zhang L. Novel 3D hexapod computer-assisted orthopaedic surgery system for closed diaphyseal fracture reduction. Int J Med Robot 2012;8:17-24.

24. Townsend G, Richards L. Twins and twinning, dentists and dentistry. AUST DENT J 1990;35:31727.

25. Du H, Hu L, Li C, Wang T, Zhao L, Li Y, et al. Advancing computer-assisted orthopaedic surgery using a hexapod device for closed diaphyseal fracture reduction. Int J Med Robot 2015;11:348-59.

26. Bai L, Yang J, Chen X, Sun Y, Li X. Medical Robotics in Bone Fracture Reduction Surgery: A Review. Sensors (Basel) 2019;19.

\section{Tables}


Table1 General information of two groups of patients

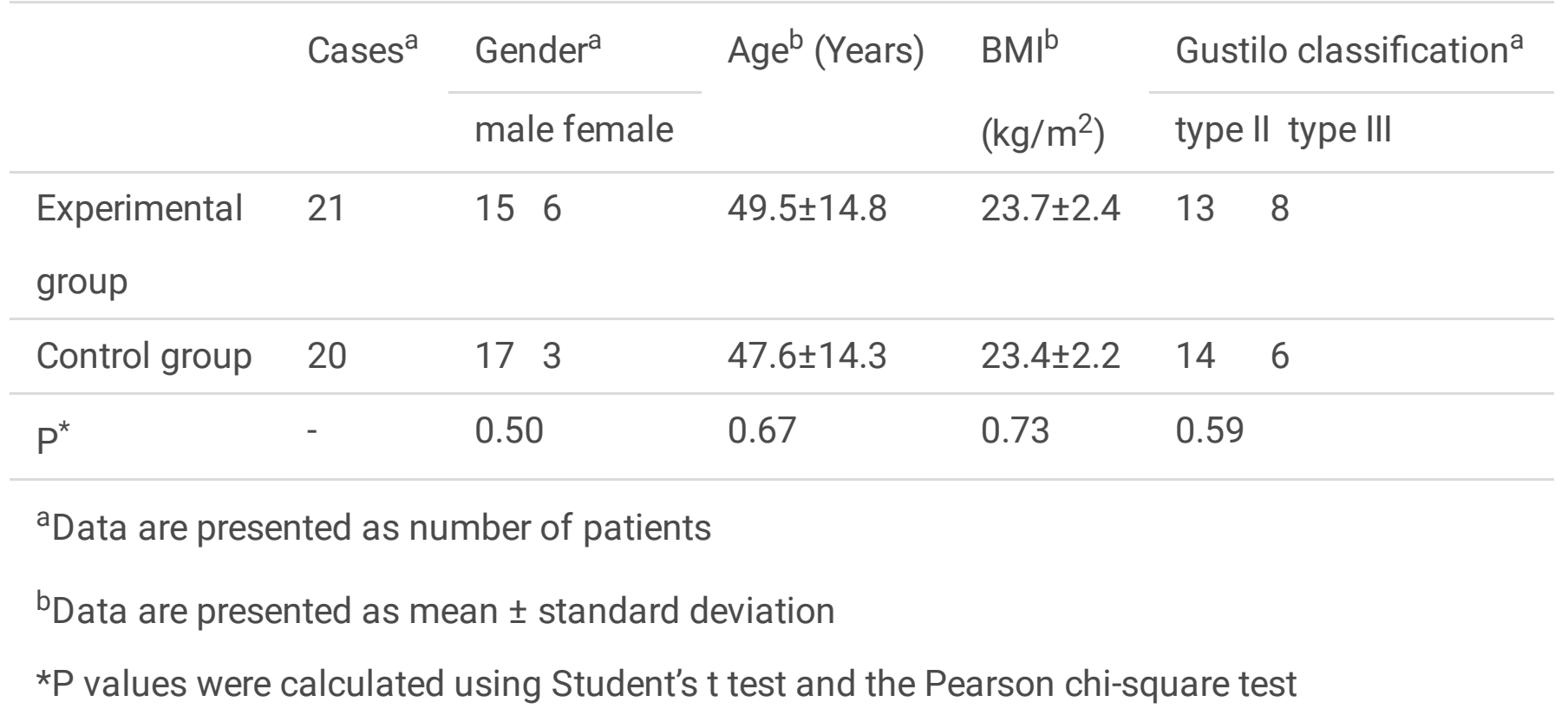

Table 2 Comparison of residual deformities between the two groups after adjusting

\begin{tabular}{llll} 
Variable & $\begin{array}{l}\text { Experimental } \\
\text { group }\end{array}$ & Control group & P value* \\
\hline $\mathrm{M} 1^{\mathrm{a}}(\mathrm{mm})$ & $0.50(0,1.72)$ & $1.74(0.43,3.67)$ & 0.024 \\
$\beta 1^{\mathrm{b}}\left(^{\circ}\right)$ & $0(0,1.25)$ & $1.25(0.62,1.95)$ & 0.020 \\
$\mathrm{M}^{\mathrm{a}}(\mathrm{mm})$ & $0(0,1.22)$ & $2.02(0,3.74)$ & 0.016 \\
$\beta 2^{\mathrm{b}}\left(^{\circ}\right)$ & $0(0,0)$ & $1.42(0,1.93)$ & 0.004
\end{tabular}

M1 was the distance of the inward/outward movement in AP X-ray of the distal bone fragment relative to the proximal bone fragment; $\mathrm{M} 2$ was the forward/backward movement in lateral X-ray between the two bone fragments; $\beta 1$ was the angle of varus/valgus in AP X-ray of the distal bone segment relative to the mechanical axis of the proximal bone segment; $\beta 2$ was the angle of extension/flexion in lateral X-ray between the two bone fragments.

${ }^{a}$ Data and ${ }^{b}$ Data are presented as Median (P25, P75)

*P values were calculated using Mann-Whitney U test

\section{Figures}



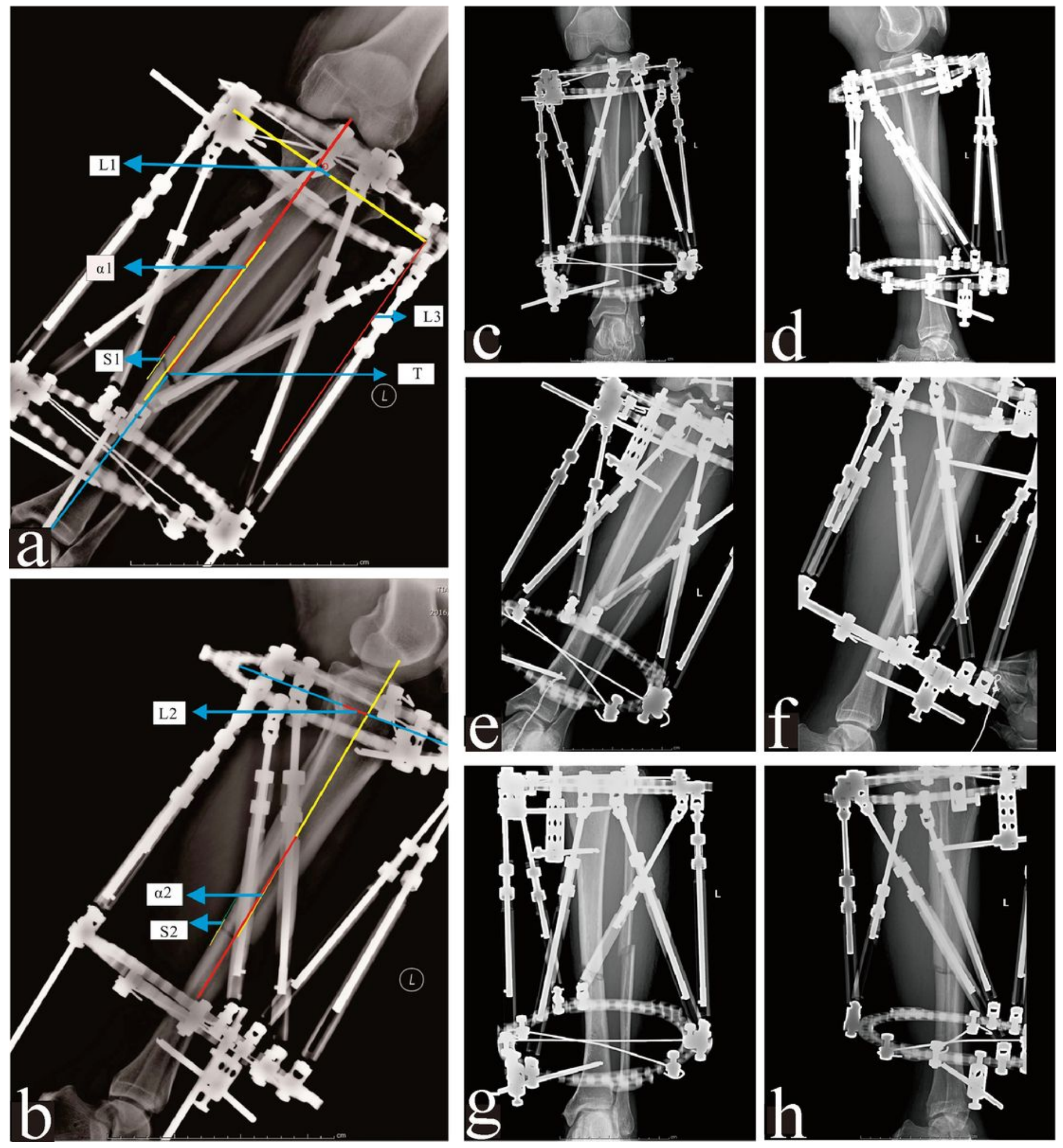

Figure 1

Radiographs showing patient with tibiofibular fracture treated with TSF using traditional radiograph measurement method. Male,58 years old, left side. a Measuring deformity parameter in AP radiograph. b Measuring deformity parameters in lateral radiograph. c AP radiograph after adjusting. d Lateral radiograph after adjusting. e 1 month after surgery of AP radiograph. $f 1$ month after surgery of lateral radiograph. g 3 months after surgery of AP radiograph. h 3 months after surgery of lateral radiograph. 


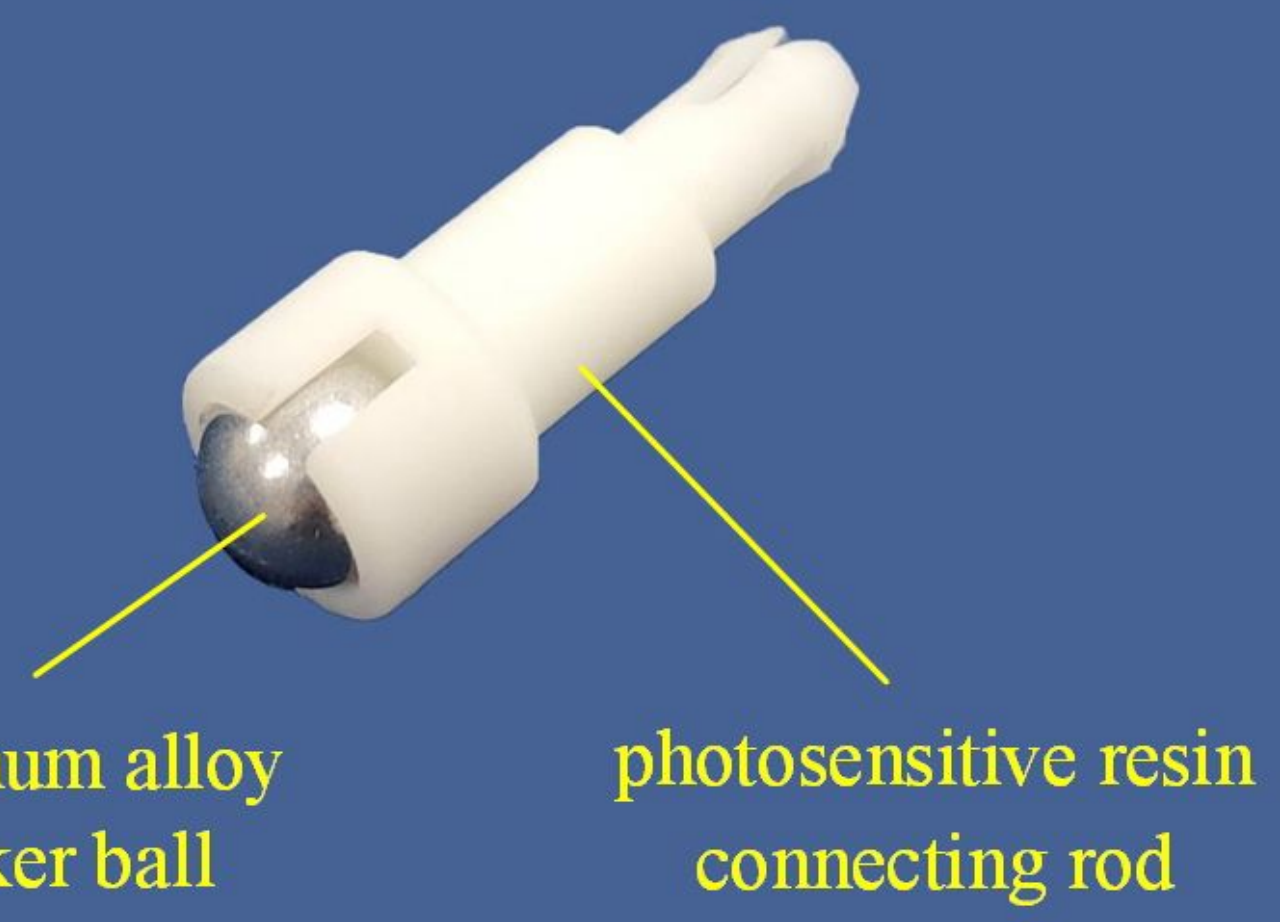

Figure 2

The composition of the marker 

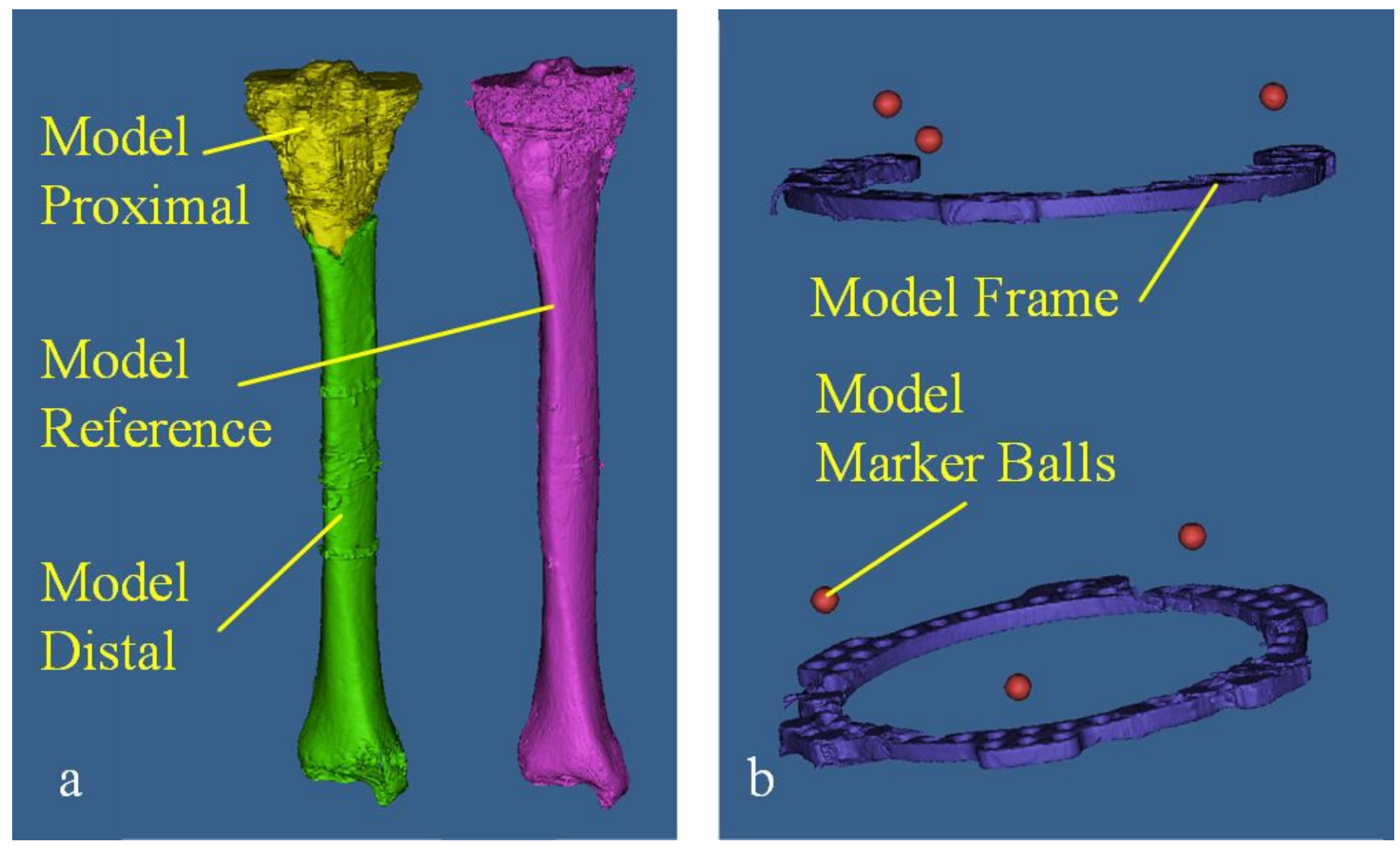

\section{Figure 3}

The 3D model of reconstruction. The fracture line was used as the boundary to divide the reconstructed affected limb bone model into the proximal and distal bone model. a Model Proximal (the 3D model of the proximal bone of the affected limb), Model Distal (the 3D model of the distal bone of the affected limb) and Model Reference (the 3D mirror model of healthy limb bone). b Model Frame (the 3D model of external fixation), and Model Marker Balls (the 3D model of Marker Balls). 


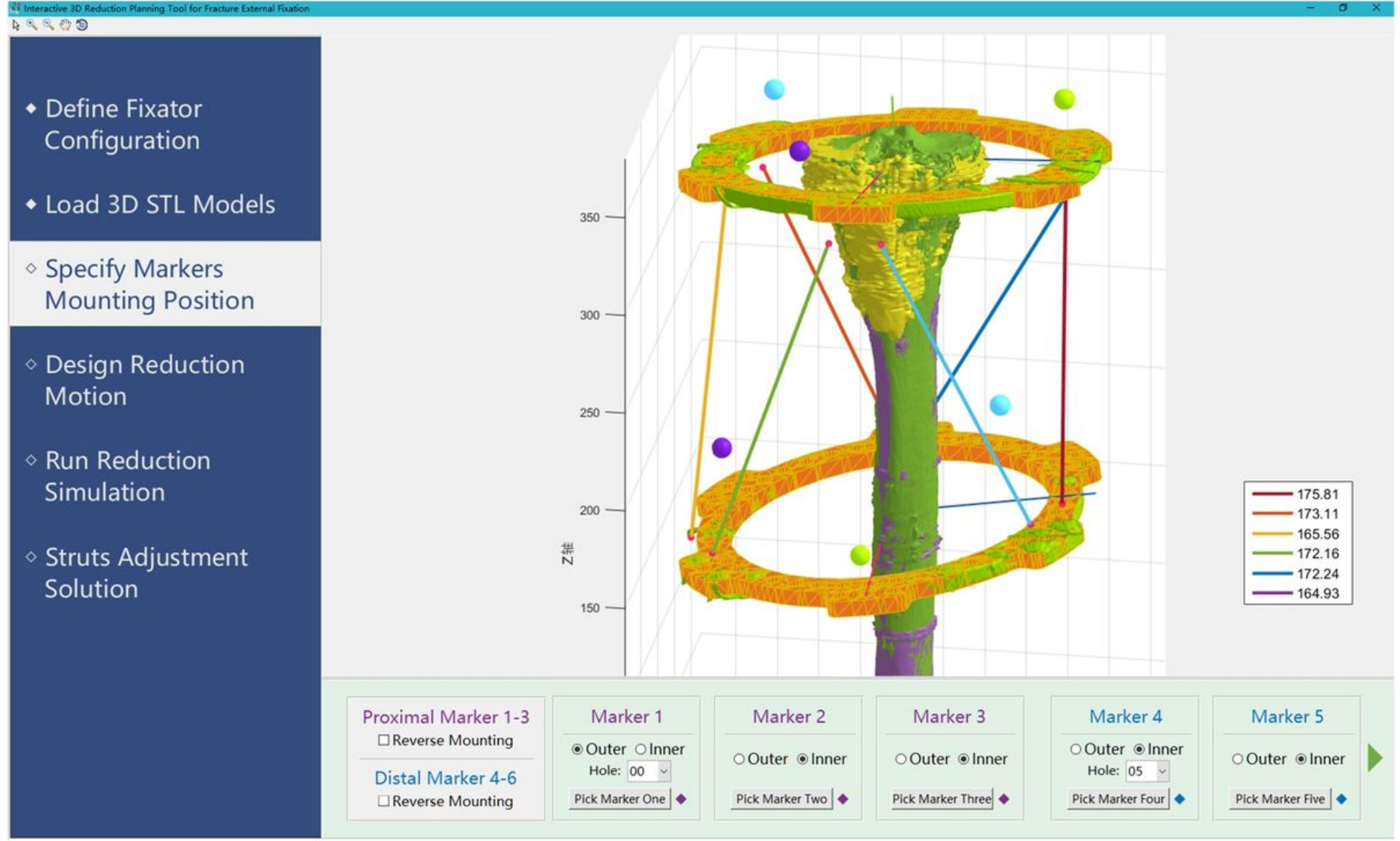

Figure 4

The interface of the 3D reduction software 


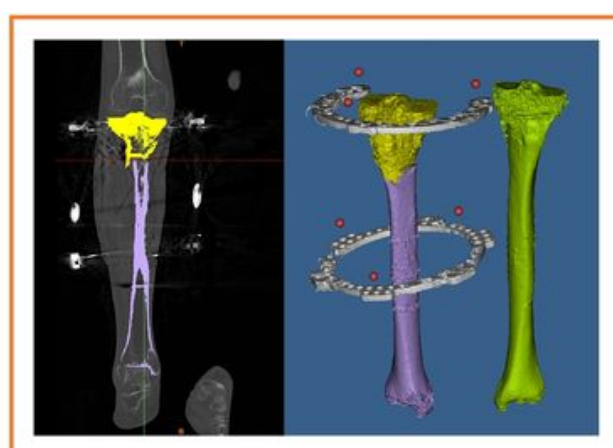

Prep. step: 3D reconstruction and model segmentation

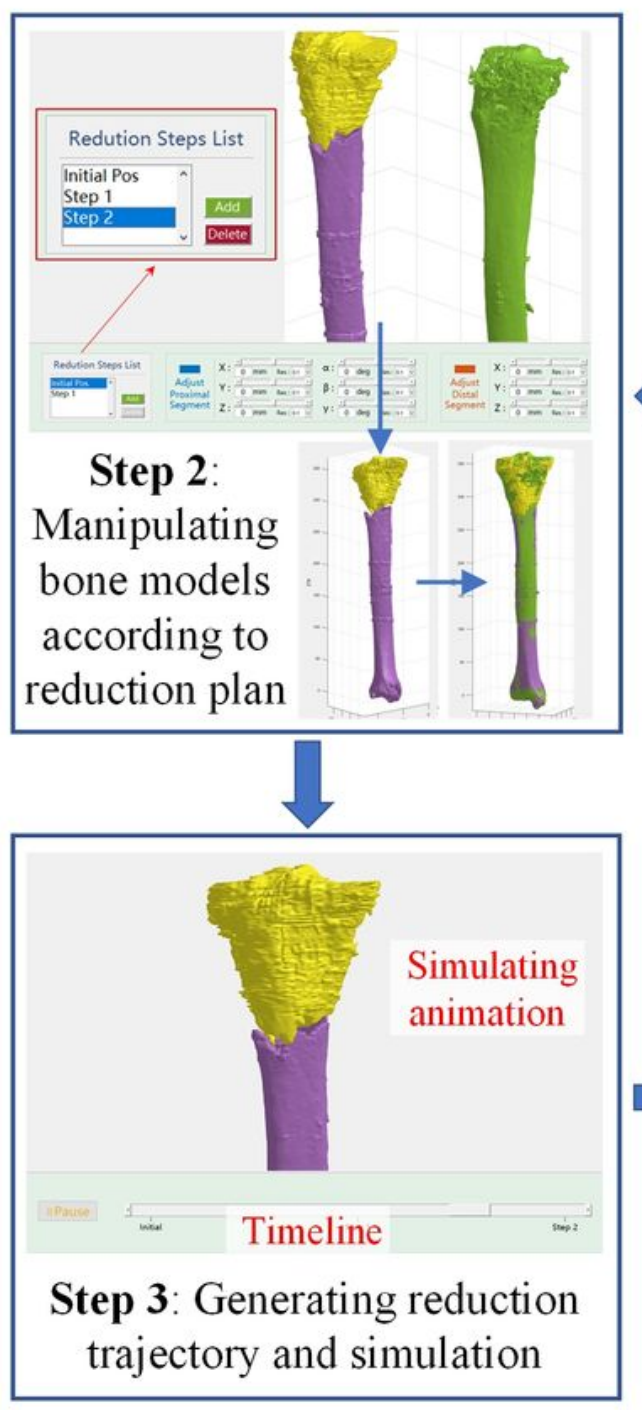

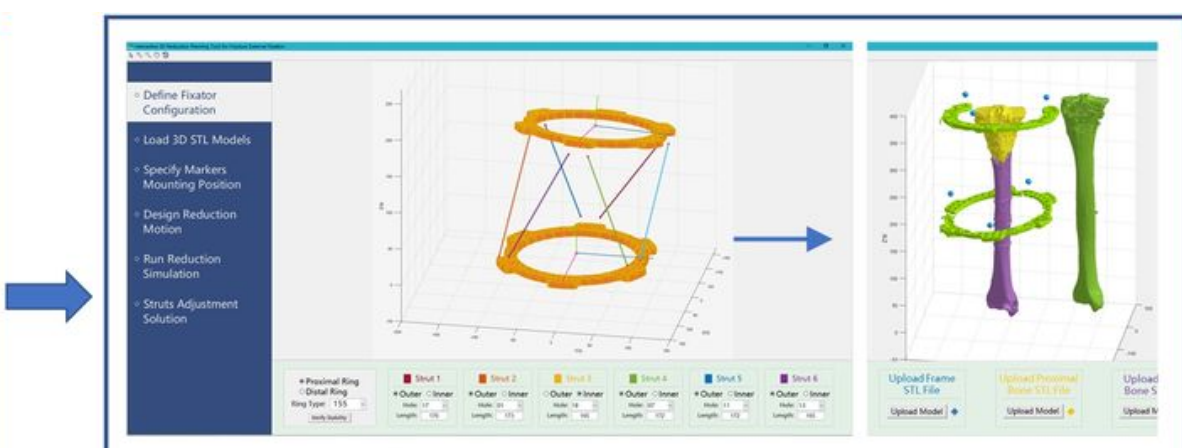

Step 1a: Inputting frame structure information and importing 3D STL files

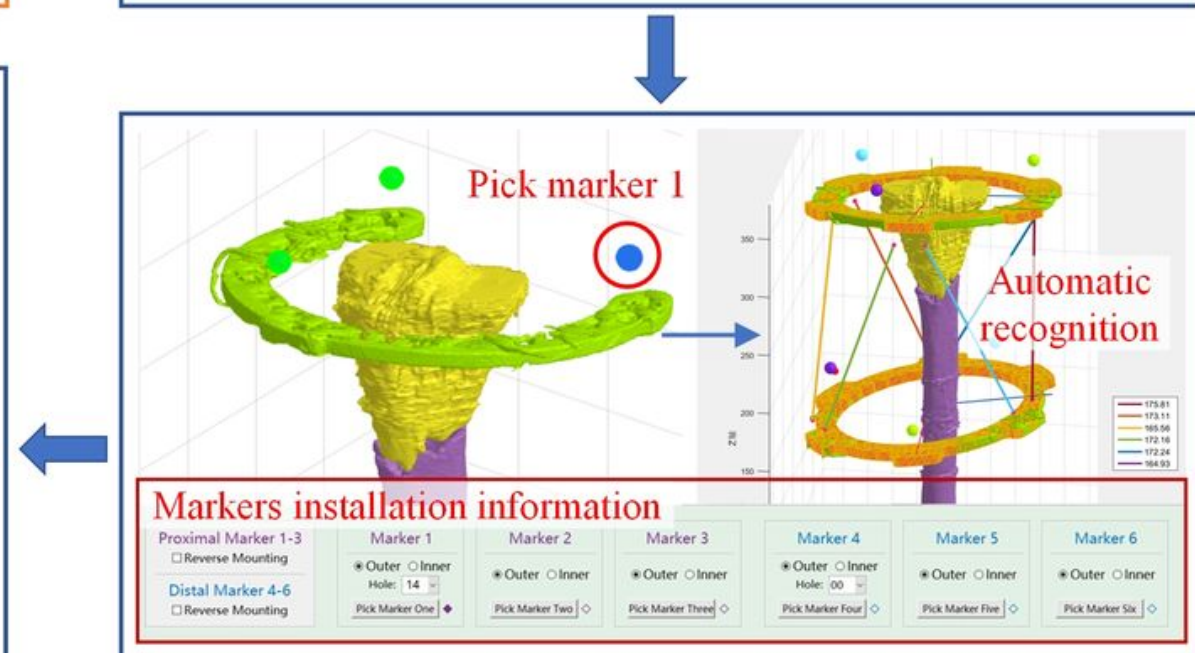

Step 1b: Specifying markers installation information and automatically recognizing position and pose parameters

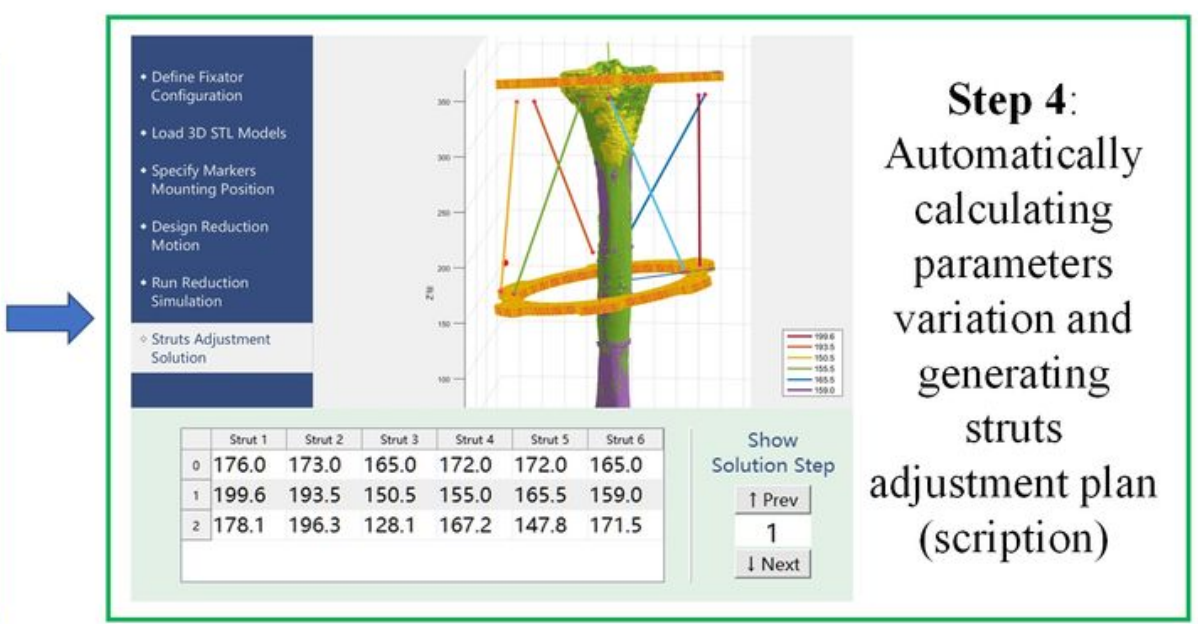

\section{Figure 5}

Schematic diagram of the marker-3D reconstruction method 

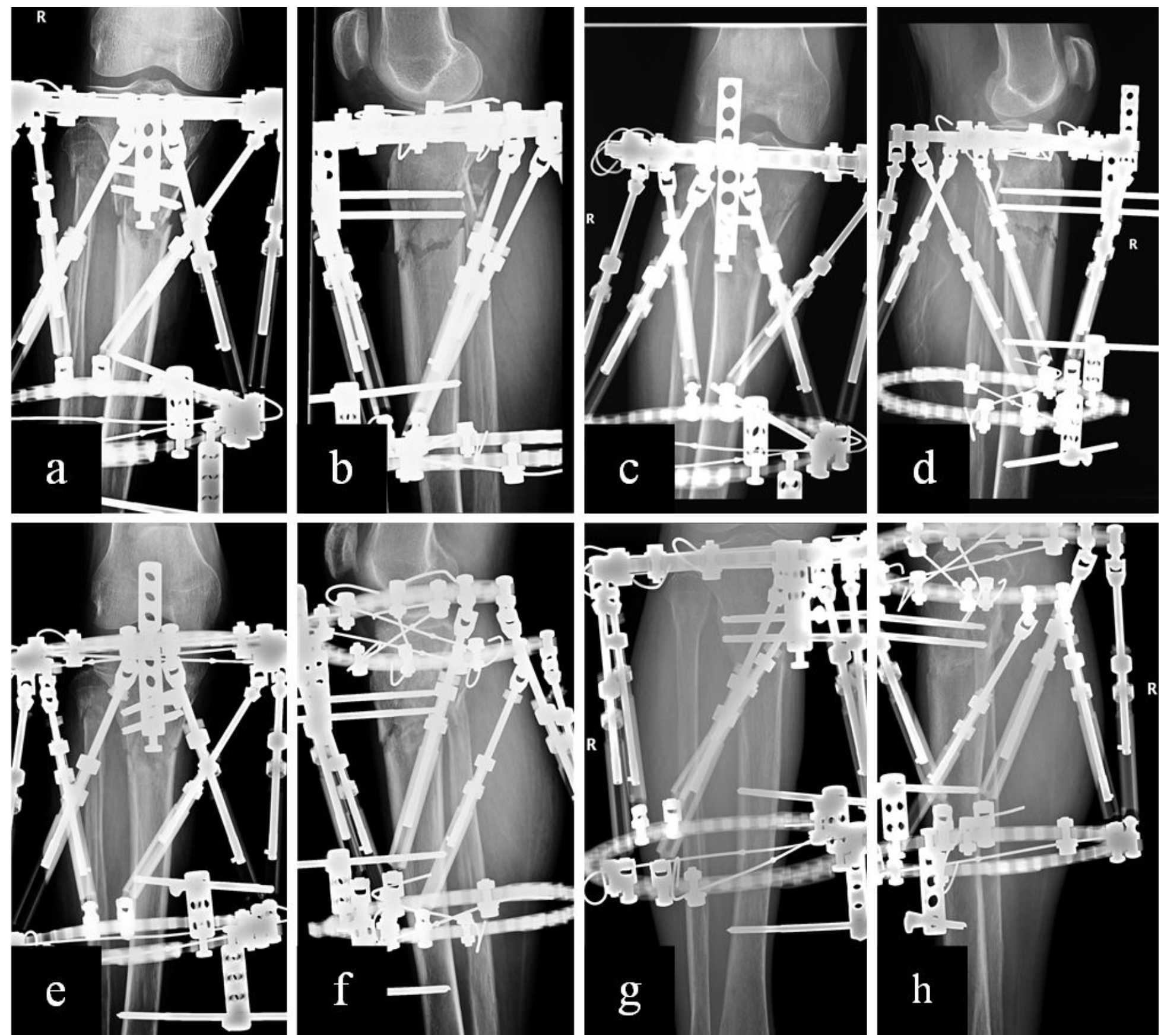

Figure 6

Radiographs showing patient with tibiofibular fracture treated with TSF using marker-3D measurement method. Male,74 years old, right side. a AP radiograph after surgery. b Lateral radiograph after surgery. c AP radiograph after adjusting. $d$ Lateral radiograph after adjusting. e 1 month after surgery of AP radiograph. $\mathrm{f} 1$ month after surgery of lateral radiograph. g 3 months after surgery of AP radiograph. $\mathrm{h} 3$ months after surgery of lateral radiograph 